UDC 627.11:627.81

SCOPUS CODE 1900

https://doi.org/10.36073/1512-0996-2019-4-66-74

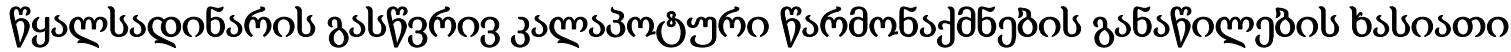

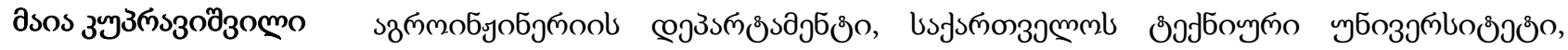

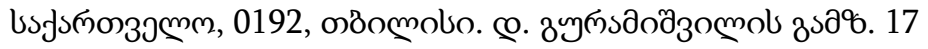

E-mail: m.kupravishvili@gtu.ge

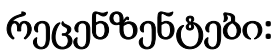

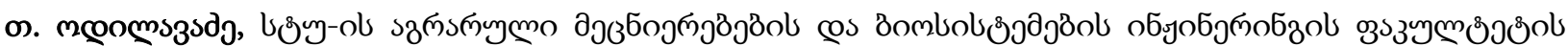

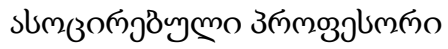

E-mail: adilavadze2004@yahoo.com

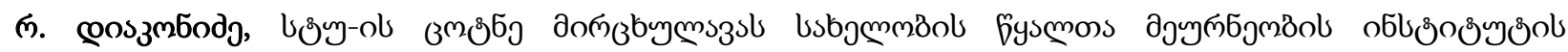

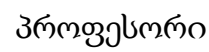

E-mail: robertdia@mail.ru

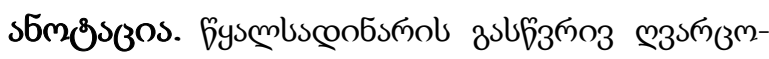

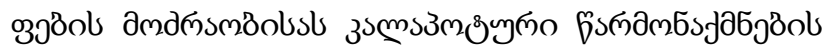

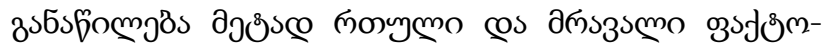

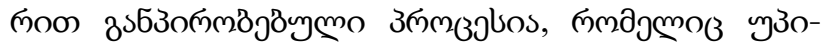

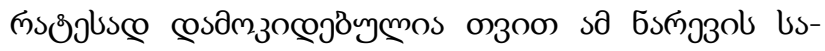

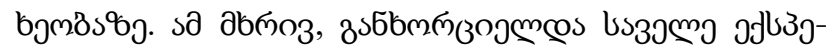

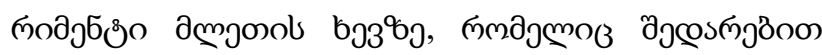

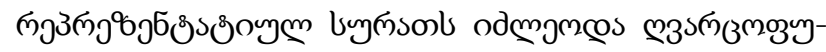

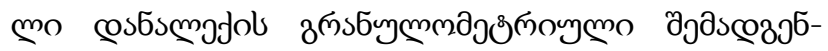

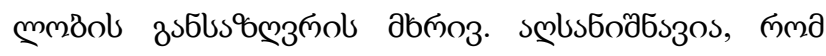

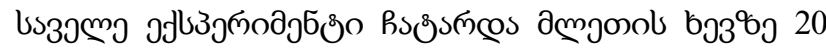

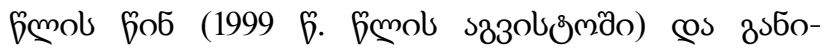

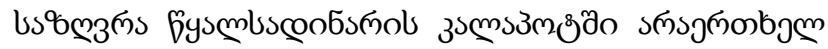

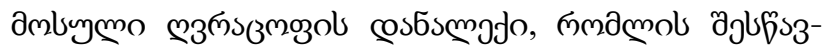

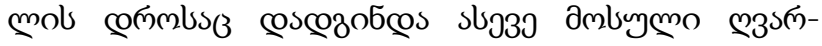

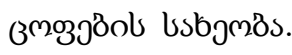

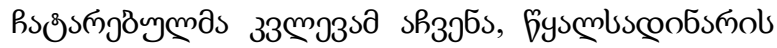

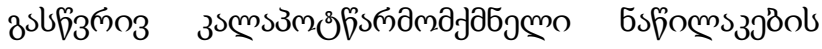

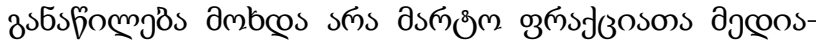

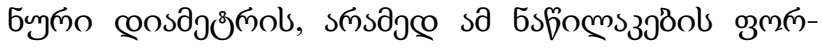

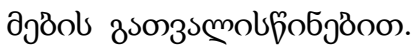

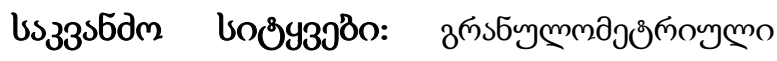

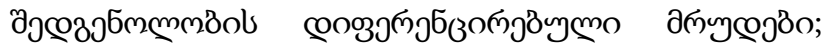

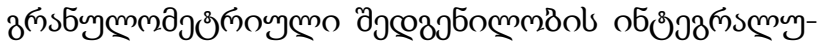

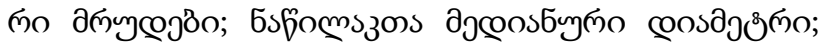

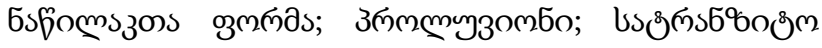
ómbs. 


\section{gjlsszsmo}

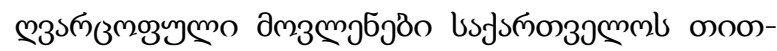

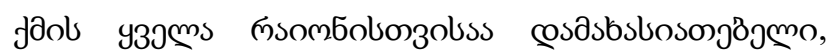

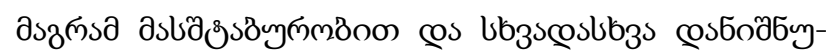

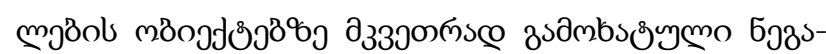

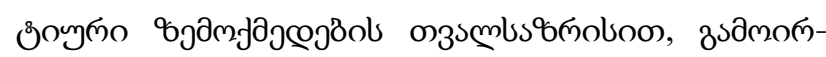

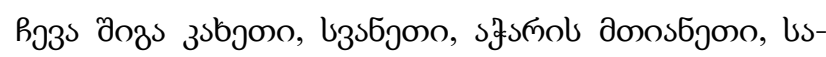

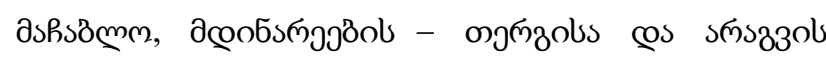

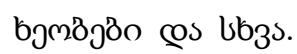

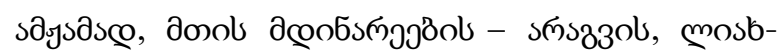

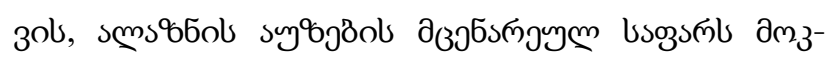

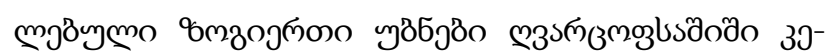

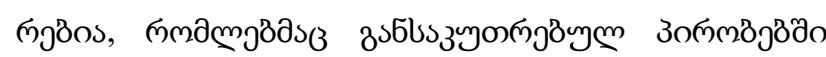

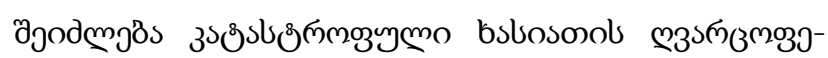

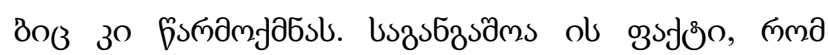

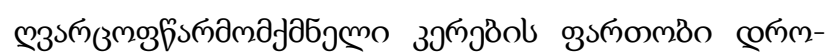

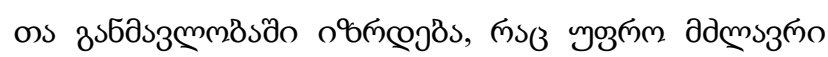

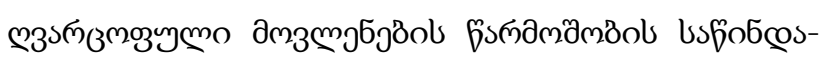

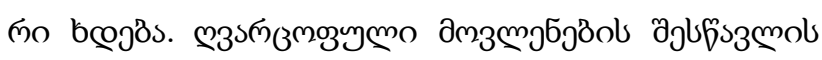

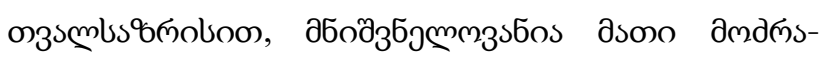

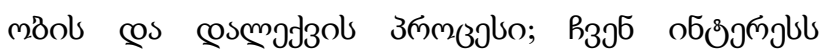

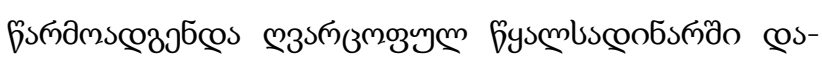

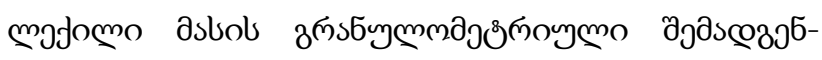

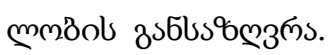

\section{domoosseo 5sfomo}

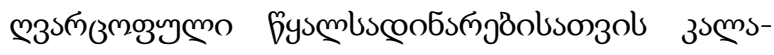

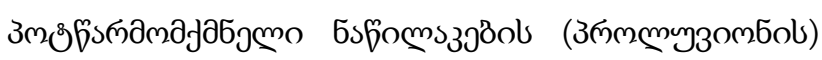

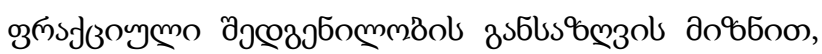

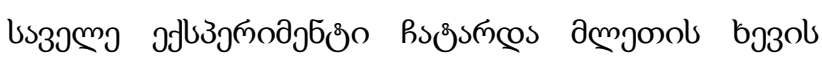

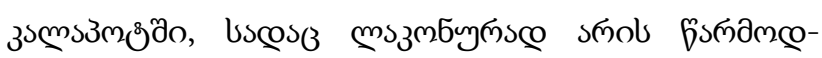

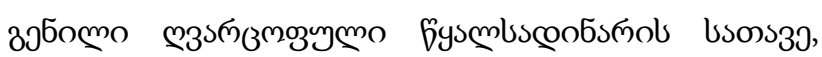

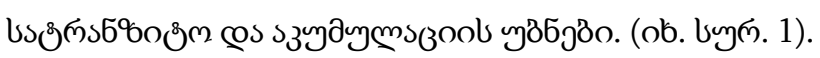

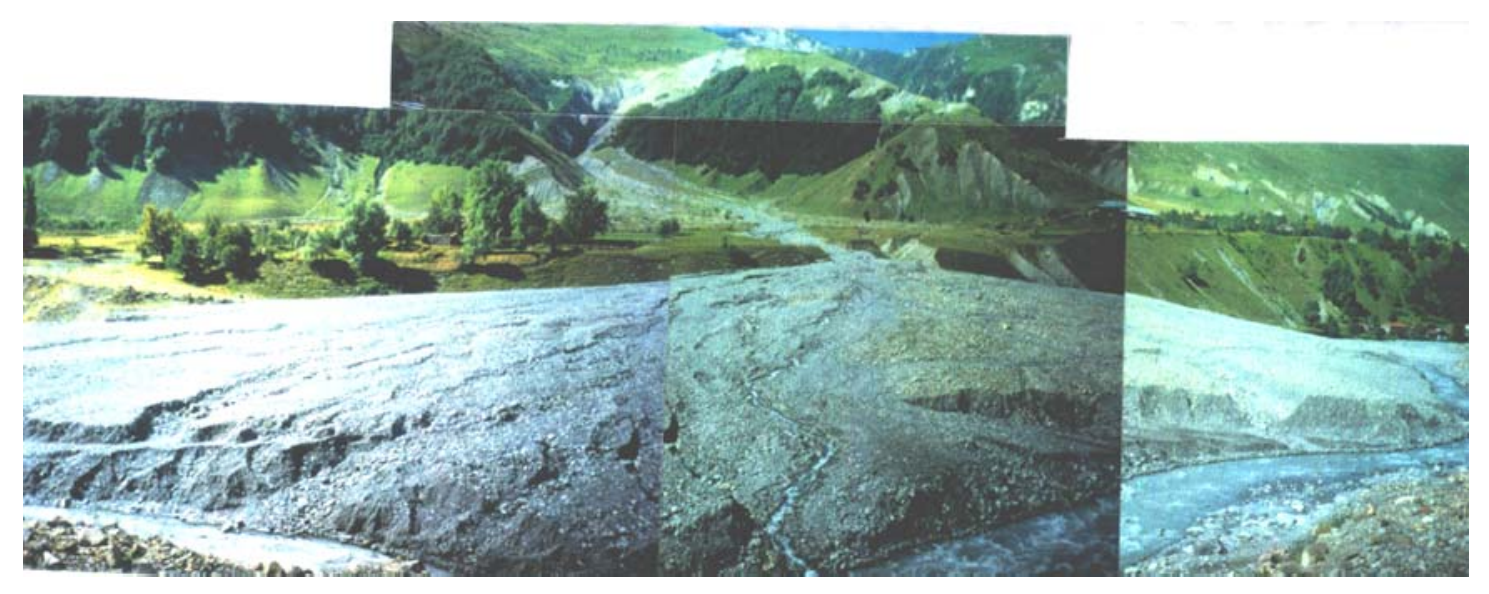

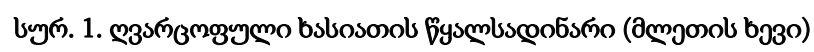

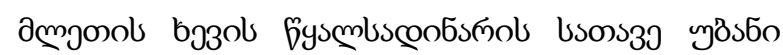

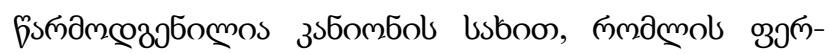

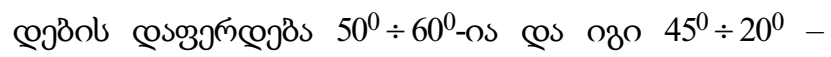

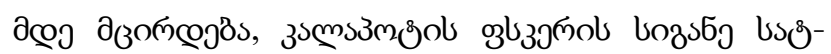

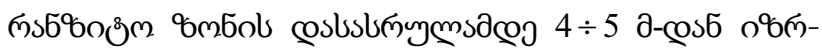

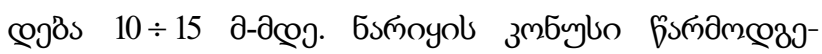

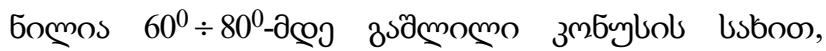

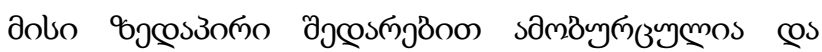




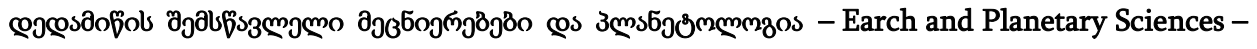
Науки изучающие Землю и Планетология

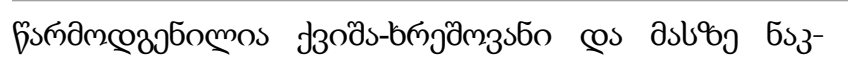

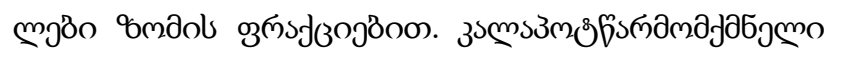

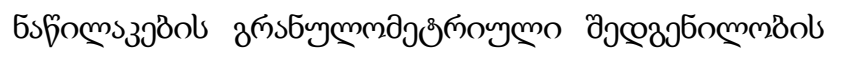

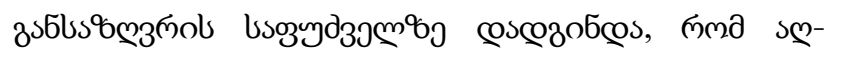

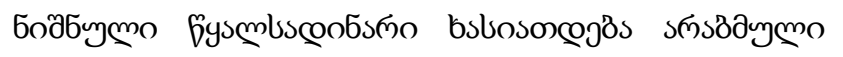

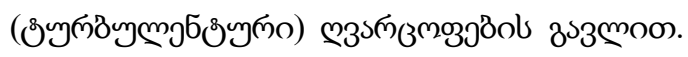

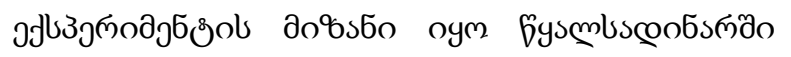

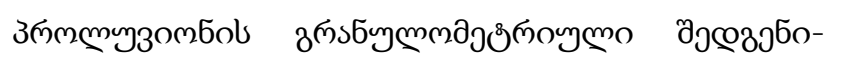

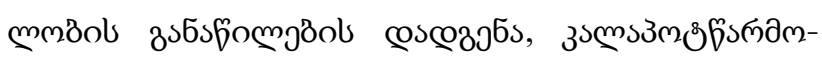

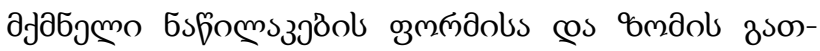

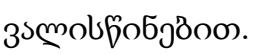

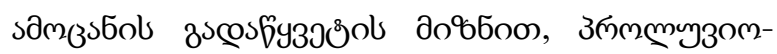

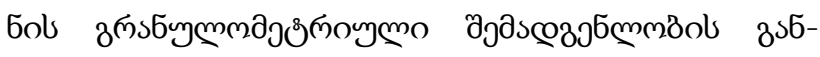

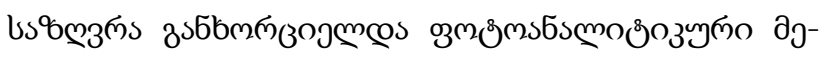

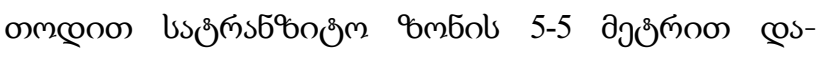

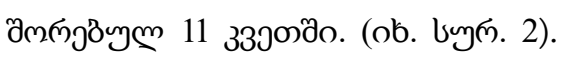

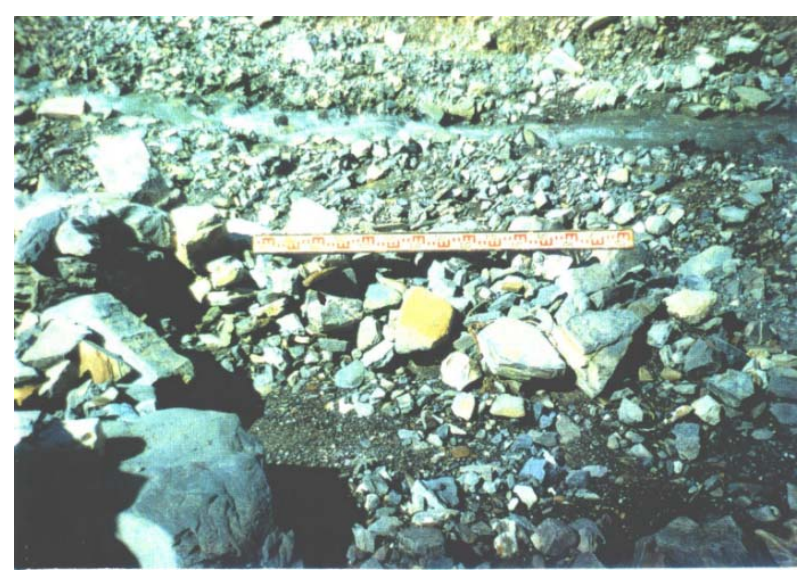

s)

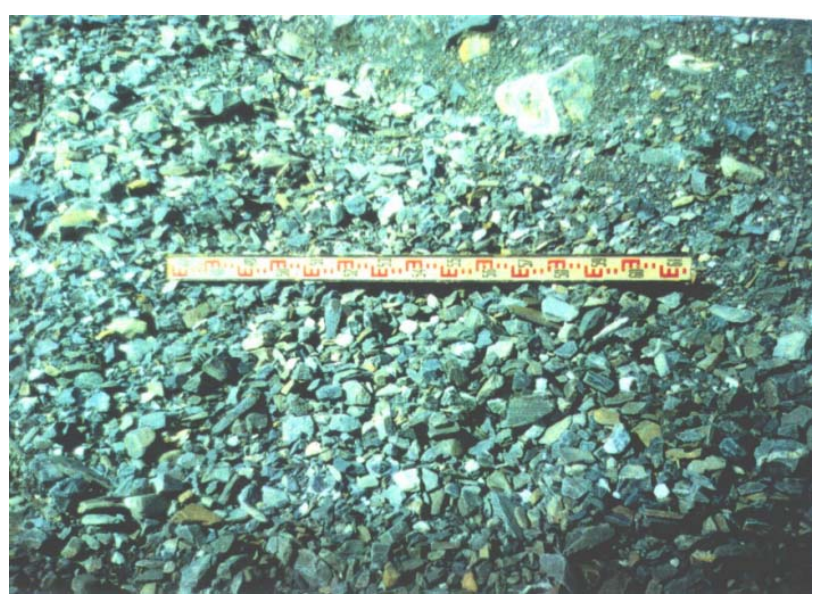

8)

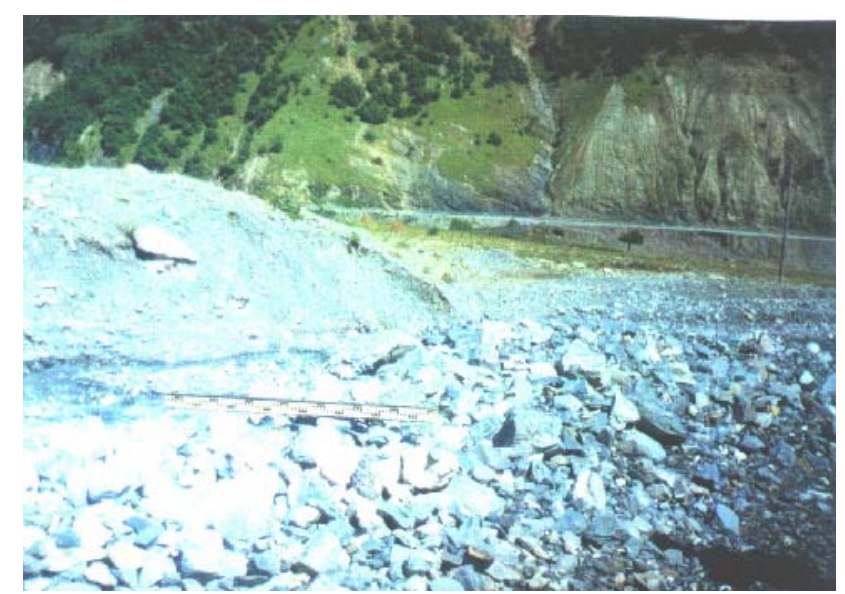

3)

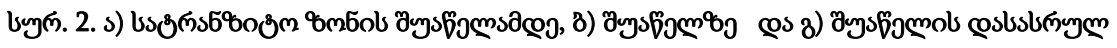

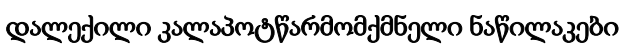




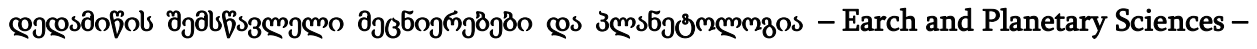
Науки изучающие Землю и Планетология

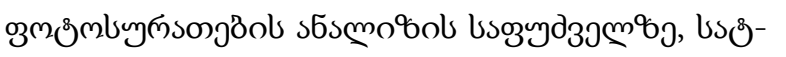

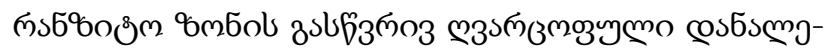

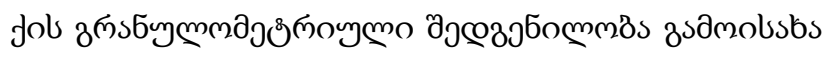

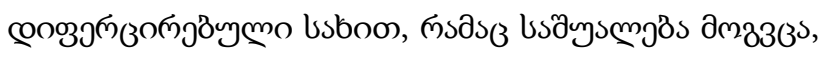

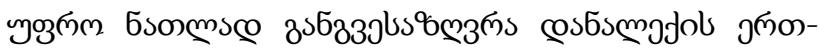

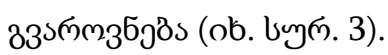

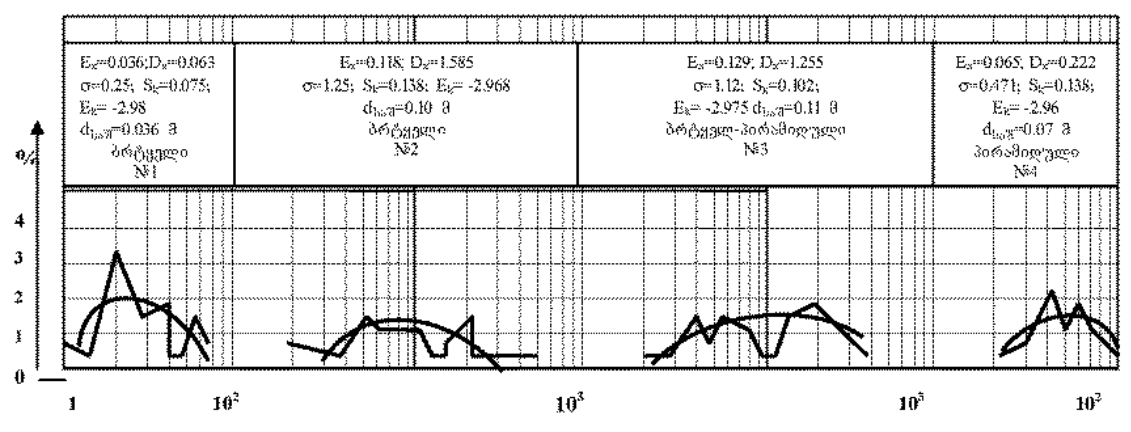

s

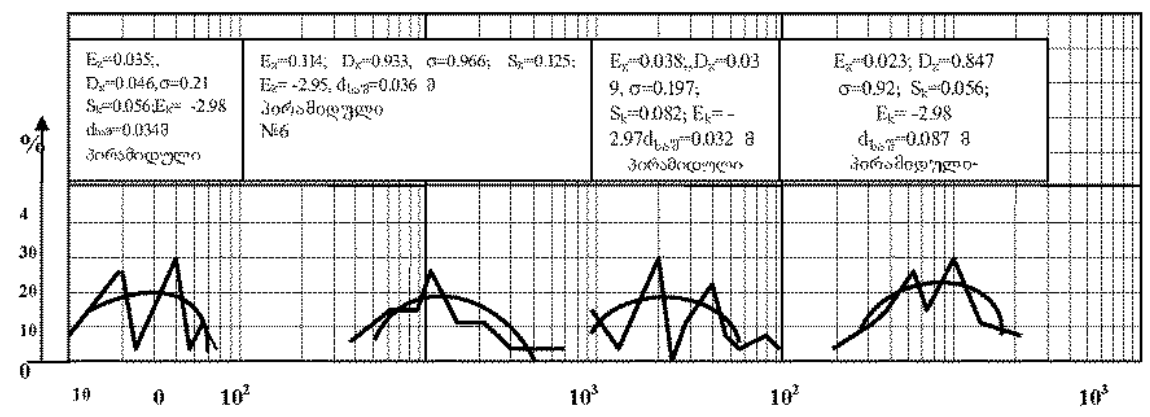

8

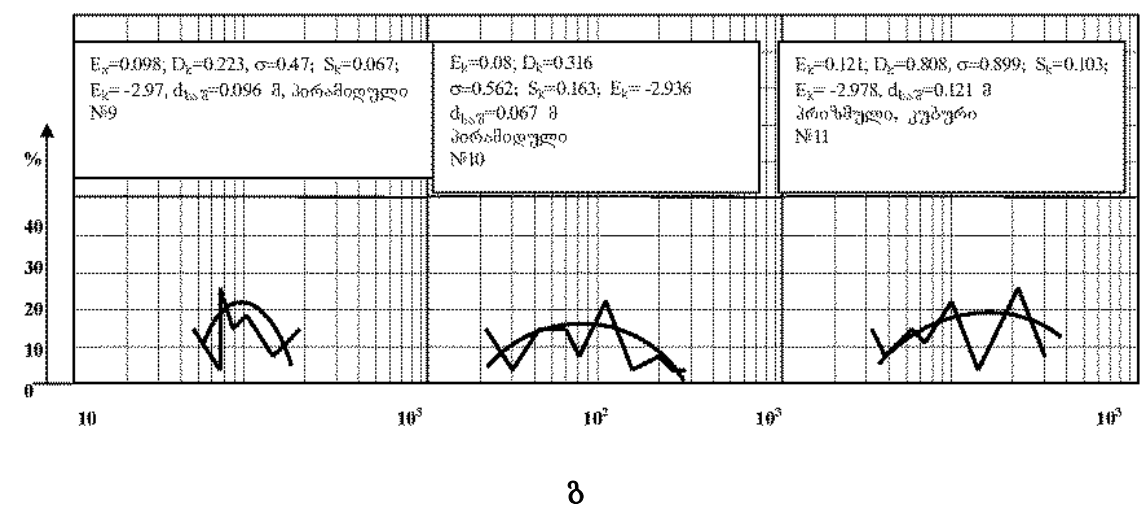

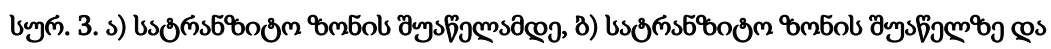

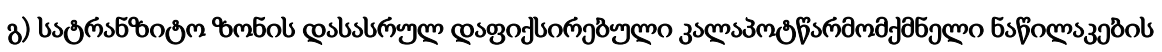

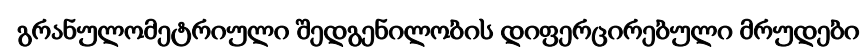

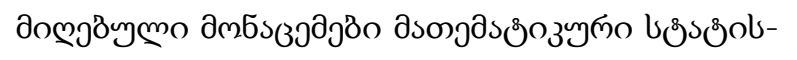

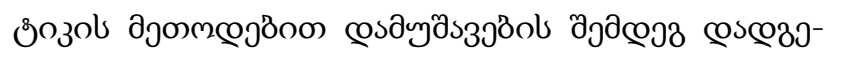

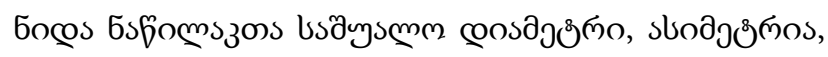

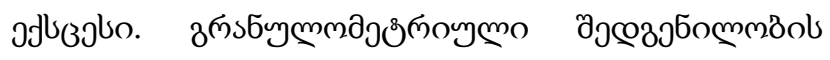

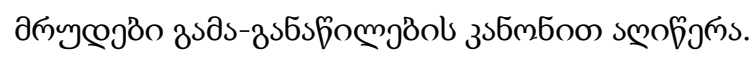




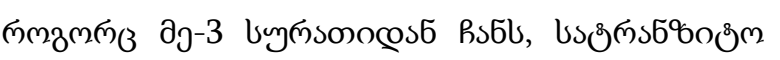

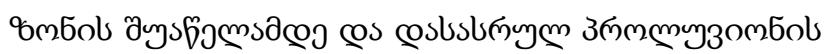

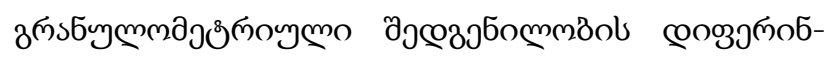

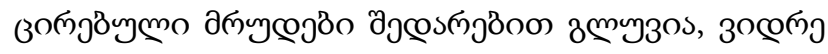

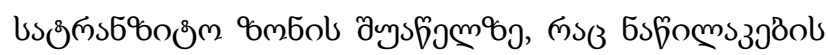
ј๓о口з

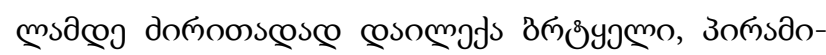

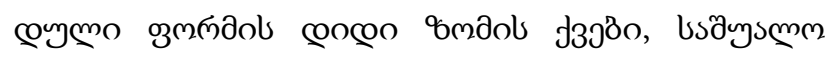

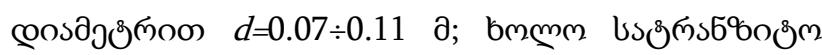

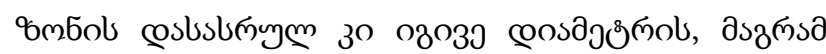

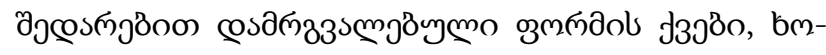

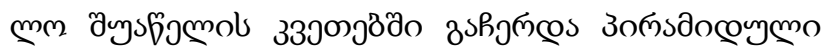

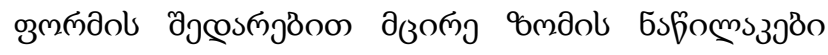
$(d=0.032 \div 0.087$ d).

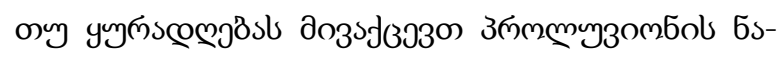

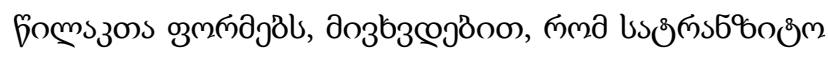

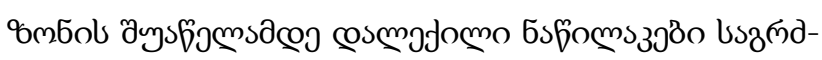

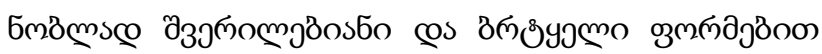

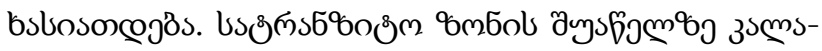

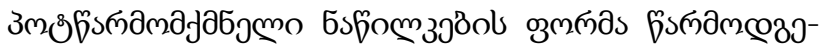

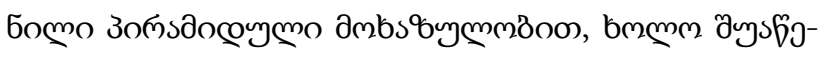

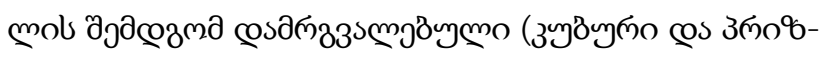

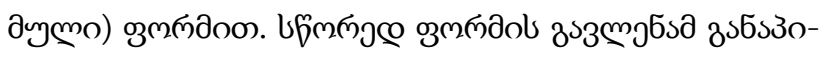

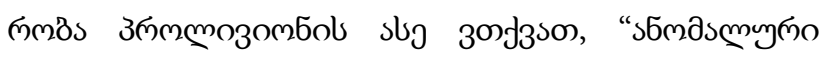

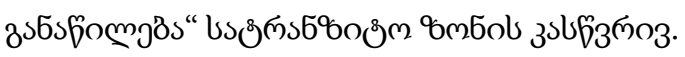

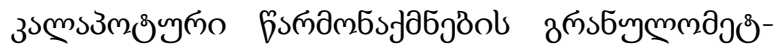

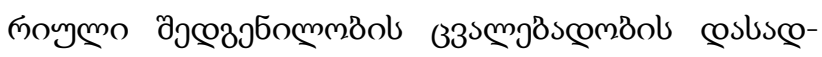

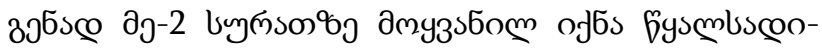

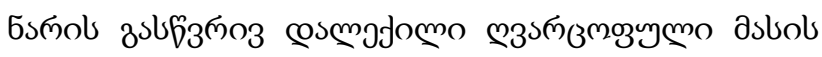

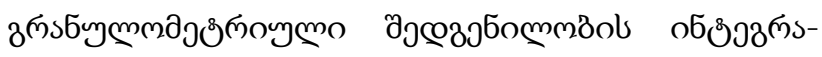
милюо дпумјво.

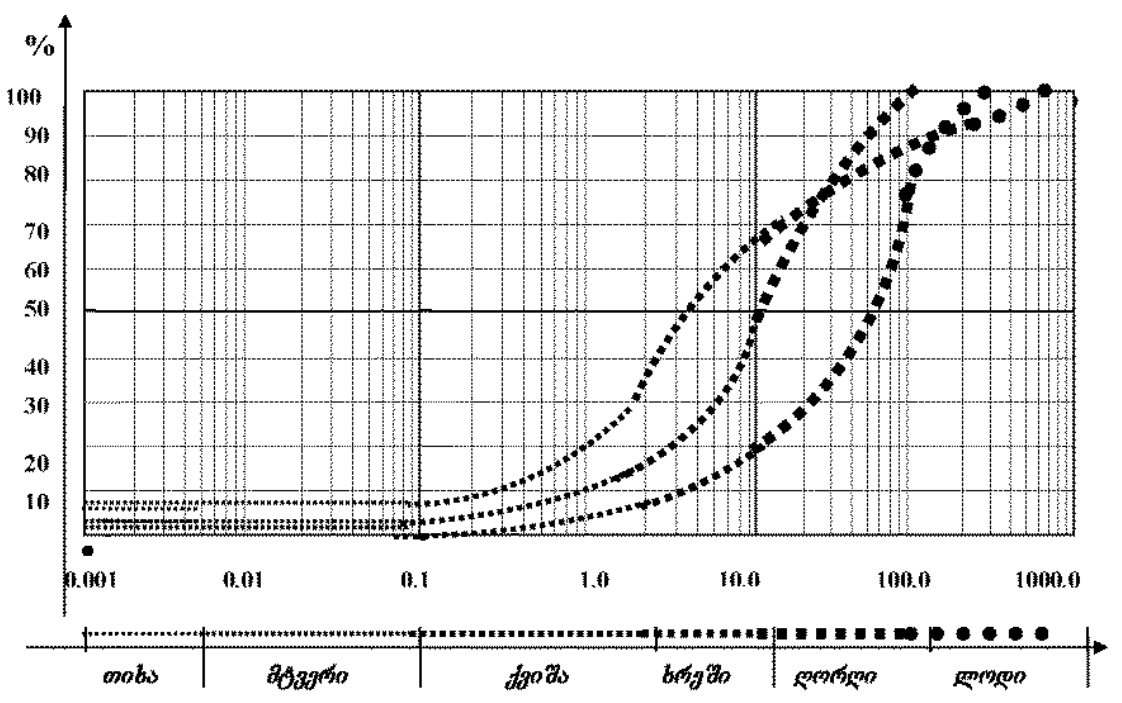

d, 1 a

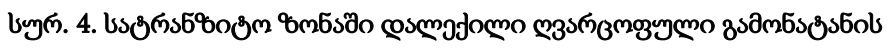

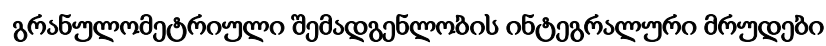

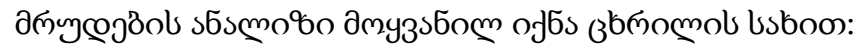




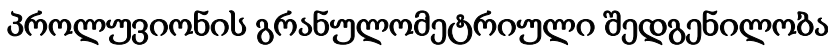

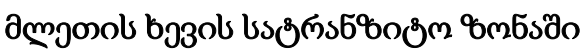

\begin{tabular}{|c|c|c|c|c|c|}
\hline \multirow{2}{*}{ 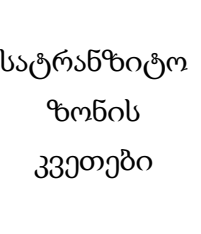 } & \multicolumn{5}{|c|}{ 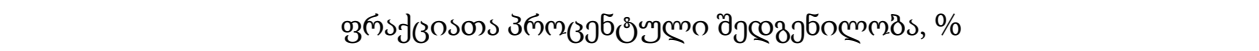 } \\
\hline & 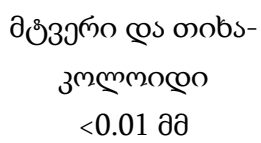 & $\begin{array}{c}\text { J3oð̀ } \\
0.01-2.0 \text { да }\end{array}$ & 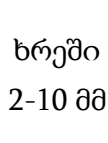 & $\begin{array}{l}\text { ммпুо } \\
\text { 10-200 да }\end{array}$ & $\begin{array}{c}\text { ммсуда } \\
200<\text { да }\end{array}$ \\
\hline 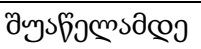 & 5 & 25 & 35 & 25 & 10 \\
\hline 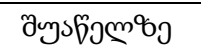 & 5 & 10 & 30 & 55 & - \\
\hline cosbsliñym & 3 & 5 & 15 & 70 & 7 \\
\hline
\end{tabular}

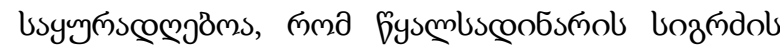

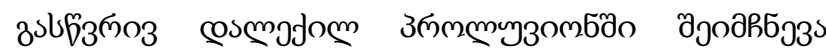

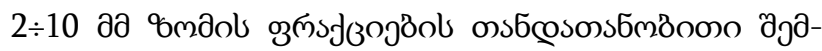

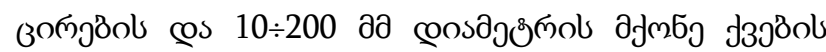

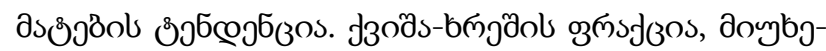

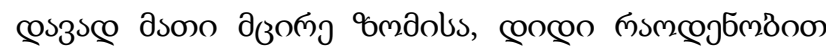

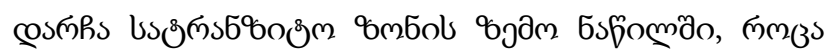

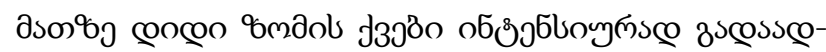

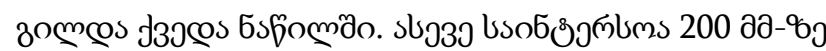

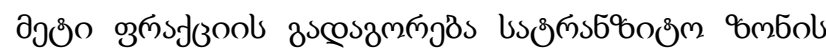

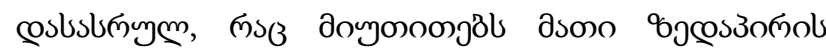

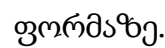

\section{cosb $33^{6 s}$}

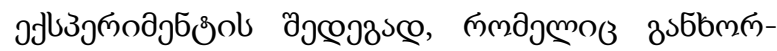

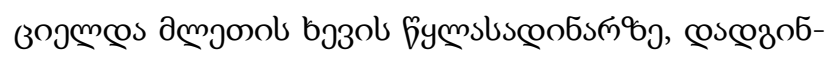

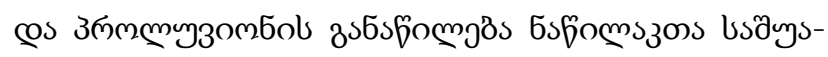

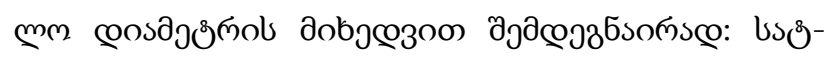

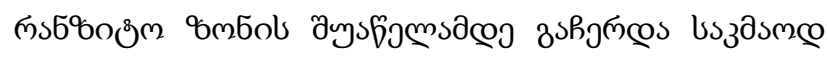

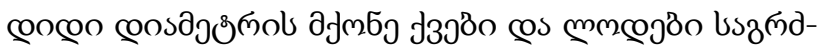

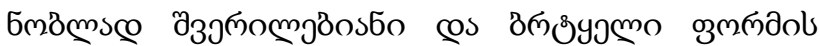

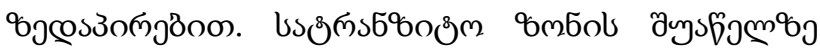

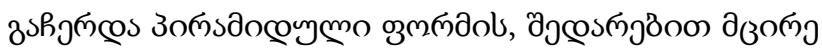

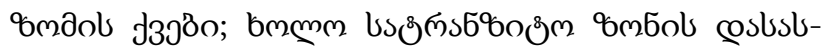

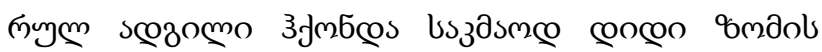

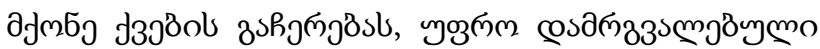

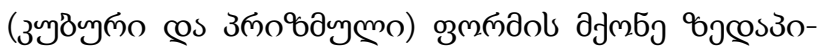

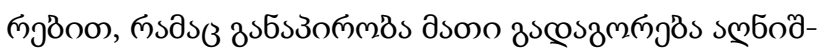

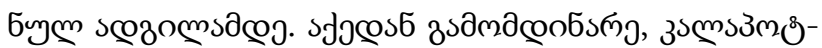

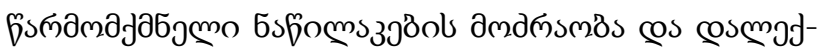

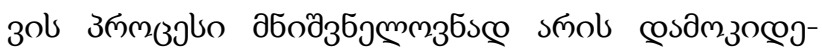

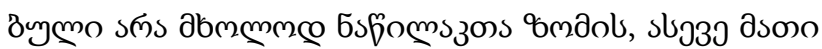

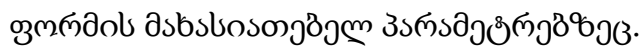

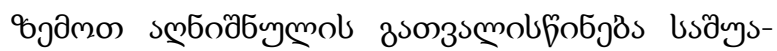

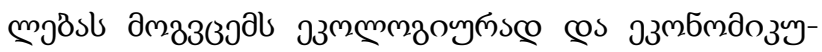

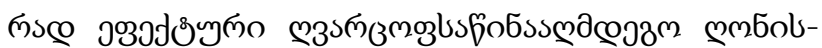

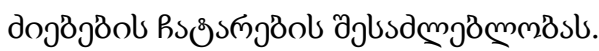




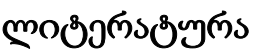

1. Kruashvili I., Kukhalashvili E., Inashvili I. Influence of morphometrical and hydraulic characteristics on the form of cross-section of a channel. Annals of agrarian science. Vol. 7. №2. 2009, 114-116 pp.

2. Kruashvili I, Loiscandl W., Inashvili I., Bziava K., Himmelbauer M. Debris flow channel processes and determination of the lateral compression ratio. 5th international conference "Debris flows: Disasters, risk, forecast, protection". Tbilisi. 2018, 643-649 pp.

3. Kruashvili I., Kukhalashvili E., Inashvili I., Bziava K., Lortkipanidze D. Determining the properties of hyperconcentrated flow. International journal of innovative research in science, engineering and technology (IJIRSET). Vol. 5. Issue 11. 2016, 6 p.

4. Kruashvili I.G., Kukhalashvili E.G., Inashvili I.D., Bziava K.G. Debris flow phenomena. Risk, Prediction, Protection. monografia. saqartvelos teqnikuri universiteti, tbilis, 2017, 249 gv.(in Georgian).

5. Natishvili O.G., Kruashvili I.G. Deceived infrastructure objects from mudflows. (Environmental issues). (L.A.P. lambert Academic Publishing, Germania, 2016, 104 st. (in Russian).

6. Natishvili O.G., Kruashvili I.G., Inashvili I.D. Applied problems of the dynamics of connected mudflows. Monografia, OOO “Nauchtexlituzdat" Moskva, 2018, 144 st. (in Russian).

7. Natishvili O.G., Kruashvili I.G. Protection of objects from the harmful effects of mudflows. J. Ekologicheskie sistemi I pribori, \#7, 2015, st.20-30. (in Russian).

8. Sheko A.I. Methods of studying and predicting of exogenous geological processes. M. 1988, st. 215 (in Russian)

9. Takahashi T. Debris flow. Taylor and Francis Group. London,UK, 2007, 465 p. (in English).

10. Ventcel E.S., Ovcharov L.A. Applied problems of probability theory. M. "Radio I sviaz".1983, st.414 (in Russian). 
UDC 627.11:627.81

SCOPUS CODE 1900

\title{
The distribution of channel formations (proluvium) along the watercourse
}

\author{
Maia Kupravishvili Department of Agro-Engineering, Georgian Technical University, 17 D. Guramishvili \\ Str, 0192, Tbilisi, Georgia \\ E-mail: m.kupravishvili@gtu.ge
}

\section{Reviewers:}

T. Odilavadze, Associate Professor, Faculty of Agricultural Science and Biosystems Engineering, GTU

E-mail: adilavadze2004@yahoo.com

R. Diakonidze, Professor, Tsotne Mirtskhulava Water Management Institute of the Georgian Technical University

E-mail: robertdia@mail.ru

\footnotetext{
Abstract. As a result of experiment conducted on the watercourse Mletis-Khevi, proluvium distribution was determined by the average particle diameter as follows: stones and boulders with large diameters, ledges and flat surface shapes had stopped at the middle of the transit zone meanwhile fractions of relatively smaller diameter with pyramid shapes had stopped in the middle of the transit zone and large stones with rounded surfaces were noted at the end of the transit zone.

Thus, the motion and sedimentation of the sedimentary particles depend not only on the size (diameter) but also on the shape of the particles.

Taking into account above mentioned will enable us to conduct ecologically and economically effective mudflow measures.
}

Key words: Differential curves of granulometric composition; integral curves of granulometric composition; median particle diameter; particle shape; proluvium; transit zone. 
UDC 627.11:627.81

SCOPUS CODE 1900

\section{Характер распределения русловых образований (пролювиона) вдоль водотока}

Мая Куправишвили Департамент Агроинженерии, Грузинский Технический Университет, Грузия, 0192, Тбилиси. пр. Д. Гурамишвили 17

E-mail: m.kupravishvili@gtu.ge

\section{Рецензенты:}

Т. Одилавадзе, ассоциированный профессор факультета аграрных наук и инженеринга биосистем ГТУ

E-mail: adilavadze2004@yahoo.com

Р. Диаконидзе, профессор водного хозяйства Института Цотне Мирцхулава ГТУ

E-mail: robertaia@mail.ru

Аннотация. В результате эксперимента, проведенного на водотоке Млетис-Хеви, распределение пролювиона было определено по среднему диаметру частиц следующим образом: до середины транзитной зоны остановились камни и валуны с большими диаметрами, с выступами и плоскими формами поверхности, в середине транзитной зоны остановились фракции относительно меньше диаметром пирамидальных форм, а в конце транзитной зоны были отмечены большие камни с округлёнными поверхностями.

Таким образом, движение и седиментация осадочных частиц существенно зависит не только от размера (диаметра), но и от их форм частиц.

Предусмотрение вышеуказанного даст возможность проводить экономически и экологически эффективные противоселевые мероприятия в транзитной зоне селевого водотока.

Ключевые слова: водоток; дифференциальные кривые гранулометрического состава; интегральные кривые гранулометрического состава; пролювион; средний диаметр частиц; транзитная зона; форма частиц. 